\title{
Community-made mobile videos as a mechanism for maternal, newborn and child health education in rural Uganda; a qualitative evaluation
}

\author{
Juliet Ntuulo Mutanda ${ }^{1}$, Peter Waiswa ${ }^{2}$, Sarah Namutamba ${ }^{2}$
}

1. Department of Epidemiology and Biostatistics, School of Public Health, Makerere University College of Health Sciences, Kampala, Uganda, Correspondence: jnmutanda@musph.ac.ug

2. Department of Health Policy Planning and Management, School of Public Health, Makerere University College of Health Sciences, Kampala, Uganda

3. Uganda Demographic Surveillance Site, Iganga District

\begin{abstract}
Introduction: In Uganda, the maternal, newborn and child mortality is highest in rural areas, which are least served by health services and are also least reached by effective behavior change communication for health. Though maternal and child health related messages are available, they are still not culture and context specific for effective behaviour change.

Aim: This study aimed at evaluating the feasibility of using locally made videos by local community groups in local languages as a channel for increasing knowledge, practices, demand and use of maternal and child health messages among women living in rural communities in Eastern Uganda.

Methods: This paper describes the qualitative findings from a quasi experimental study targeting the rural semi-illiterate populations in hard to reach areas. Videos were developed and implemented based on Ministry of Health. Focus group discussions and KIs targeted pregnant and post natal mothers. Data transcription and content analysis was done.

Results: Local mobile community videos were effective in communicating knowledge about key maternal and child health messages to both women and their male partners.

Conclusion: Locally made mobile community videos are effective in improving knowledge, attitudes, practices and use of maternal and child health messages among rural semi-illiterate communities.

Keywords: Community-made mobile videos, maternal, newborn, child health education, rural Uganda, a qualitative evaluation DOI: http://dx.doi.org/10.4314/ahs.v16i4.6

Cite as: Mutanda JN, Waiswa P, Namutamba S. Community-made Mobile Videos as a mechanism for maternal newborn and child health education in rural Uganda; a qualitative evaluation. Afri Health Sci 2016;16(4): 923-928. bttp:/ / dx.doi.org/10.4314/ abs.v16i4.6
\end{abstract}

\section{Introduction}

Africa experiences $57.8 \%$ maternal related deaths out of the 350,000 deaths experienced globally ${ }^{1}$. Sub-Saharan Africa still faces major challenges despite the global progress to fight maternal and child morbidity/mortality ${ }^{2}$. The World Health Organization defines maternal newborn and child health, as the health of mothers, infants, children and adolescents. Some of the most important

\section{Corresponding author: \\ Juliet Ntuulo Mutanda, Department of Epidemiology and Biostatistics, School of Public Health, Makerere University College of Health Sciences, Kampala, Uganda, Telephone: +256-414-543-872 Email: jnmutanda@musph.ac.ug}

areas of concern in $\mathrm{MCH}$ are maternal, newborn and child mortality and morbidity, pregnancy related complications, pre-natal and post-natal care, immunization, family planning, care of the newborn among others ${ }^{3}$. It is evident that Sub Saharan Africa is still behind in achieving the recently launched Sustainable Development Goals that seek to reduce maternal, newborn and child health ${ }^{3}$ among others.

According to the Uganda Health Sector Strategic plan II, maternal and child health, $(\mathrm{MCH})$ conditions carry the highest (435 deaths per 100,000 live births) total burden despite the progress in improvement of the health of mothers and children. Uganda is implementing the Uganda Minimum Health Care Package which primarily aims at increasing awareness and promoting community participation in health care delivery and utilization through pro-

African Health Sciences Vol 16 Issue 4, December, 2016 
moting health, preventing diseases and community health initiatives ${ }^{4}$. One of the ways of doing this is through the Village Health Teams (VHTs) strategy which also aims at providing the much needed health education. According to the Health Sector Strategic Plan II, VHT structures exist in $80 \%$ of the districts in Uganda; however, only $31 \%$ of the existing VHTs are functional. Similar to most developing countries, in Uganda, the burden of maternal and child health morbidities and mortality is highest in rural areas which are least served by health services and are also least reached by effective behavior change communication for health ${ }^{5}$. Though the maternal, newborn and child health related information is available, it is not culture and context specific and is rarely delivered in a sustainable manner. A lot of knowledge and scientifically proven health interventions such as antenatal/postnatal care is not effectively utilized. Much of this information is not well present to the targeted populations who need it most and face the highest disease burden. We believe that communities are more likely to learn and benefit from this information if it is context specific ${ }^{6}$ i.e. messages are delivered by locals, in the local languages, and these messages can be repeated and accessed any time.

It is known that disparities in maternal knowledge, attitudes and behavior negatively impacts on mother and child survival within prenatal, pregnancy and post natal periods ${ }^{7}$, leading to poor health outcomes ${ }^{8}$. A number of community based interventions such as home visits and videotaping have been effective in promoting good behavior and practices". Such interventions if coupled with effective referral systems can lead to better maternal and child health related outcomes ${ }^{10}$ and can contribute to better policies to avert maternal and child mortality in rural areas ${ }^{11}$. Community based cost effective interventions aimed at improving knowledge and health seeking behaviors are needed in the fight against maternal and child related morbidity and mortality ${ }^{5}$. Behavior change communication is defined as the process of any health intervention with communities to develop communication messages that in turn promote positive behaviors which are appropriate to their settings. Behavioral change communication messages need to be repackaged to attract such populations for positive health outcomes in developing populations ${ }^{12}$. It is believed that positive behavior change communication is more effective if its culture specific $^{6}$. The use of women groups to effect community based interventions has been proven to be effective in increasing community acceptability of health related interventions ${ }^{13}$. Advancing behavior change communication strategies to collaborate with the local communities is critical in improving health outcomes ${ }^{14}$. This has proven to be one of the most successful behavior change communications need to engage participants at a social level for better impact ${ }^{15}$. Furthermore behavior change communication strategies need to be culturally appropriate for better health outcomes ${ }^{16}$. In light of this and more, we evaluated the feasibility of using locally made mobile videos by local community groups in local languages as a mechanism for behavior change to improve maternal, newborn and child health among rural communities in Eastern Uganda.

\section{Methods}

Study area: Iganga and Luuka districts are located in Eastern Uganda, $160 \mathrm{~km}$ from Kampala district with a population of over 500,000 people and two ofthe distances with highest fertility rates in Uganda. A total of four sub-counties were engaged in this evaluation. Two of these were intervention sub-counties and two control sub-counties located far from the intervention subcounties in Luuka and Iganga districts. The sub counties were purposively selected with support from the District health teams based on availability of village health teams.

\section{Study design:}

This study employed a quasi experimental study design, with intervention and control areas based on the health belief model for behaviour change. However, this paper presents qualitative findings from the end term evaluation. The process of producing the video messages was entirely based on Ministry of Health approved maternal, newborn and child health related messages that are currently being used by village health teams during house visits to educate mothers. The videos were produced collaboratively with representatives from the Ministry of Health, Global Health Media project and a representative from World Health Organisation in charge of community health. A set of video scenes about the danger signs in newborn babies, produced by Global health media project, was interpreted into the local language and included. The videos were then produced from the communities 
and with the community health representatives participating actively. Video production process engaged the local communities throughout and actors were local people who used their local language so as to create ownership, ease mobilization of communities to watch the videos and to increase the effect of the videos on the communities.

Pre-testing of the videos was done in an area other than the intervention areas until the final set of videos was approved by the village health teams and stakeholders/ partners as one that reflects the exact messages to be communicated to the target population.

The Final videos were then shown consistently to the pregnant and post natal mothers. Two intervention sub counties watched the videos whereas the control sub counties maintained the village health teams moving from one house to another educating the mothers as was the status quo. All villages in the intervention sub counties were visited within nine months of implementation. The village health team member organized and designated the place where the mothers gathered to watch the video. This was a village/community hall, a school hall/ classroom or a church. On average, every mother in the intervention villages had an opportunity to watch the video more than once. During each video show session, the mothers were given an opportunity to ask questions related to the messages or anything health related and these were responded to. At the end of the sessions, an exit interview was given to randomly selected mothers to assess knowledge, attitudes towards the messages in the videos. The videos were shown using a projector, a petrol powered generator, speakers and a large screen in an enclosed space in each of the villages within the intervention sub counties. The effectiveness of the videos was evaluated at baseline, midterm and end term based on pre determined indicators. However this paper presents qualitative findings from the end term evaluation.

\section{Study population and sample size:}

The proposed target population were the rural semi-illiterate and hard to reach communities in Luuka and Iganga districts. According to the Uganda Bureau of Statistics, majority of the rural population in Uganda is semi-illiterate with the highest levels found in Eastern Uganda. Specifically, we targeted pregnant and post natal moth- ers since these are the mothers reached by the VHT/ CHW during home visits to offer health education and secondly, the messages were designed to target this population. Purposive selection was employed in selecting the sub counties, parishes and villages. All pregnant and post natal mothers in the selected intervention sub counties were eligible to watch the video. Selection of the video participants and actors was done purposively.

\section{Data management and analysis:}

The qualitative end term evaluation at was performed in the intervention sub counties. A total of eight Focus Group Discussions, (FGDs) with 8-12 participants and eight Key Informant Interviews (KI) were conducted. The FGDs targeted mothers who were either pregnant or with a child less than six months old. The purpose was to explore the knowledge and attitudes towards the videos from the perspectives of the mothers and the village health team members. The Research team constituted individuals familiar with maternal newborn and child health concepts but also familiar with the local language. These were trained on the purpose of the intervention, the kind of intervention implemented and the purpose of the evaluation including how to apply the tool. The key informant interviews targeted village health teams in those sub-counties. A note taker, moderator and a tape recorder were used for data collection. The data was then transcribed and analyzed thematically.

\section{Ethical considerations}

The ethical review and approval process was obtained from the Makerere University School of Public Health Institutional Review Board and Uganda National Council of Science and Technology. All participants went through the informed consent process prior to video production and the subsequent evaluations.

\section{Results}

In this section, we present qualitative results from the end term evaluation. All the respondents reportedly had watched the videos. These mentioned the different sources of information concerning pre-natal and post -natal care which include radio programmes, health facility and peers. Of importance was the fact that very few mothers receive $\mathrm{MNCH}$ messages from village health teams. And the reason given was sometimes the villages are very wide 
and some homes are so far apart, the VHTs cannot move very far. When asked about the knowledge they gained from the videos, the respondents mentioned that the videos taught them how to care for the baby, the need to visit the health facility, how to feed during pregnancy and during breast feeding. When asked to elaborate, respondents were happy to talk about the nutritional foods such as eating greens, bananas, avocados, greens and others.

They also reportedly learnt that it is important to visit the health facility for ANC and what to prepare for before you give birth. When asked to talk more about the video content, the mothers mentioned that they learnt how to care for the baby such as have cotton clothes to wrap them in and keep the baby warm, how to take care of the cord and most importantly how to tell that the new born baby is very sick as quoted;

"We were shown the danger signs and that we should visit a health facility when we get any of those signs".

The women who watched the videos mentioned that they saw a lot in the videos such as the requirements needed to prepare for delivery for the mother and the baby.

Some of the mothers added that they watched that when pregnant you need to have some money for transport to the health facility in addition to identifying danger signs like convulsion, abnormal pains and bleeding so you have to visit the health facility for assistance. The respondents also appreciated the benefits of delivering with professional assistance as quoted;

"After delivery the nurse comes back and sees how you care for the cord and also advises you on breast feeding and to bring back the child for check up".

Furthermore the respondents reportedly appreciated the care offered to small babies as shown in the videos. This included the Kangaroo Mother Care (KMC) and the possibility of squeezing breast milk for the small babies who may not be able to suckle as quoted;

"I also saw how you place the child in your chest to get warmth especially very small babies".

The respondents interviewed were also able to mention some of the birth preparedness items that the pregnant mother should have such as gloves, threads, razor blade among others.

Also important to note was that the video was very clear and that the VHTs who were speaking in the language they could understand made it easier as quoted;

"It was very clear and well interpreted".
"What was taught in the video was good".

The mothers also appreciated the fact that the video taught the men what to do such as assisting the mother to do heavy work and the need to escort them to the facility. When asked about how the videos were shown, the mother mentioned that the videos were not shown to everyone especially the men so that they can learn how to assist their wives. Despite the fact that the videos came late, the respondents appreciated the venues selected for the videos shows and the fact that local people were used to act out most of the scenes. Generally, the respondents appreciated communication of such messages using videos as quoted;

"I say that we use that video because it shows exactly what you are seeing e.g. sick baby, with big head so it was very clear and good". "It was good because it taught even how to breast feed the baby. Because it came when many lacked that knowledge".

"It is good because it teaches us what we are not taught in the health facility".

As quoted below, the video taught the mothers a few practices that they were not performing well such as cord care.

"Before watching the videos, used to use powder for cleaning the cord".

When asked about Post Natal Care, (PNC) the mothers appreciated the need for PNC because it is important to take care of the body such as through monitoring the baby weight, immunization and assist the mother with services such as family planning.

Village health teams were randomly selected from the two sub counties. These were peer VHTs who are familiar with the community videos and had an opportunity to watch them. Prior to introducing the local videos to communicate $\mathrm{MNCH}$ messages in this area, the village health teams used to deliver this information to the mothers through door to door house visits in the villages, during burial meetings and during any spontaneous meetings in the villages. In some instances, the mothers would look for the VHTs in case they needed any health related information. The VHTs reportedly experience a number of challenges such as failure to easily identify the pregnant women, long distances to their homes, and heavy workload since the VHTs are not supported to do this. In addition, sometimes their partners do not readily welcome the VHTs into their homes. With further discussions, the 
VHTs revealed that despite the fact that the videos were shown few times within the villages, it is a good idea that may solve some of their challenges. The VHTs requested that this may be improved if the videos were shown consistently and the videos were shown to the husbands and the adolescents as well.

\section{Discussion}

Evidence has shown that a number of community based interventions such as home visits and videotaping have been effective in promoting good behavior and practices$^{9}$. This was a feasibility study which was implemented for at least six months and the results have shown that using context specific materials to communicate health related messages is effective especially among illiterate rural communities. This is similar to findings from related studies which have also found that well designed messages using platforms such as these are effective in communicating health related messages among populations to encourage behavior change ${ }^{17}$. More so, the outcomes from this pilot study confirm the need to design communication strategies that target specific population needs ${ }^{18}$. Similar to other countries in Africa, in Uganda, communication for behaviour change strategies have been implemented through a number of approaches such as written IEC materials, radio and television programmes among others $^{12,19}$.

However, most of these are not easily accessed by a magnitude of rural communities who present the highest burden of diseases, less education and high economic challenges ${ }^{20}$. Therefore findings from this pilot study show that mothers loved the videos and the messages were clearly understood. If scaled up, this will support communications for behaviour change. Needless to say if such effective behaviour change communication approaches are combined with other approaches, behaviour change can be achieved ${ }^{21}$. As is the current practice in rural Uganda, Village Health Teams, (VHTs) move from one home to another to deliver maternal newborn and child health messages however as mentioned in the interviews, very few mothers have been accessing these services. During this pilot, VHTs appreciated and strongly recommended the local community videos as a mechanism for improving the understanding, attitudes, demand and use of maternal newborn and child health services. The effectiveness of the local community videos was evident based on the qualitative findings that suggested that the videos were able to increase knowledge and understanding of maternal newborn and child health messages.

It is important to note that the local communities appreciated the messages from the videos and understood. maternal newborn and child health messages like how to care of the 'small' babies, the importance of visiting a health facility for antenatal, delivery and post-natal care, nutrition and much more. The mothers appreciated that they were able to visually see the danger signs of a pregnant woman and their new born babies. Furthermore the respondents reportedly appreciated the care offered to small babies as shown in the videos. This included the kangaroo mother care and the possibility of squeezing breast milk for the small babies who may not be able to suckle. These messages were important in that the women were able to visualize and interpret the messages from the community videos. As documented in the findings, the mothers appreciated the fact that the videos were delivered in the local language that they could understand and the interactive sessions gave them time to understand the concepts better.

This is similar to previous studies that have emphasized the need to provide context specific and culturally acceptable health education strategies for better health outcomes in disadvantaged populations ${ }^{22}$. We acknowledge that the videos were attracting men who were able to appreciate their role in assisting the pregnant woman before during and after birth. For the purposes of the pilot study, peer VHTs were purposively selected. Amidst the many successes achieved from this intervention, the village health team members evidently had positive experiences with the videos and strongly recommended them. This is because, given the challenges experienced with the current mode of health promotion in the rural areas; the local community videos are able to make their work more efficient and effective.

\section{Conclusion}

Locally made mobile community videos are effective in communicating maternal, newborn and child health messages and increasing knowledge, changing attitudes and improving practices such as the use of maternal newborn and child health messages among women living in rural communities for better health outcomes in Eastern Uganda. The need to scale up the local community videos , make them as available as possible across rural communities cannot be emphasized further. 


\section{Acknowledgements}

We would like to thank Grand Challenges, Canada for funding this feasibility study, the field coordinators; Josephine Naigaga and Sarah Namutamba and the communities of Eastern Uganda who participated in the videos. We declare that no conflicts of interests exist.

\section{References}

1. Sambo, L.G., J.M. Kirigia, and G. Ki-Zerbo, Perceptions and viewpoints on proceedings of the Fifteenth Assembly of Heads of State and Government of the African Union Debate on Maternal, Newborn and Child Health and Development, 25-27 July 2010, Kampala, Uganda. BMC Proc, 2011. 5 Suppl 5: p. S1.

2. Zhang, L., et al., Trends in child mortality: a prospective, population-based cohort study in a rural population in south-west Uganda. Paediatr Int Child Health, 2013.33(1): p. 23-31.

3.UNICEF, MILLENNIUM DEVELOPMENT GOALS; Improve maternal health. 2004.

4. Okuga, M., et al., Engaging community health workers in maternal and newborn care in eastern Uganda. Glob Health Action, 2015. 8: p. 23968.

5. Nabudere, H., D. Asiimwe, and R. Mijumbi, Task shifting in maternal and child health care: an evidence brief for Uganda. Int J Technol Assess Health Care, 2011. 27(2): p. 173-9.

6. Ntshebe,O., J.M. Pitso, and A.K. Segobye, The use of culturally themed HIV messages and their implications for future behaviour change communication campaigns: the case of Botswana. SAHARAJ, 2006. 3(2): p. 466-76. 7. Beck, L.F., et al., Prevalence of selected maternal behaviors and experiences, Pregnancy Risk Assessment Monitoring System (PRAMS), 1999. MMWR Surveill Summ, 2002. 51(2): p. 1-27.

8. Suellentrop K, M.B., Williams L, Monitoring progress toward achieving Maternal and Infant Healthy People 2010 objectives--19 states, Pregnancy Risk Assessment Monitoring System (PRAMS), 2000-2003. 2006.

9. Black, Home and videotape intervention delays early complementary feeding among adolescent mothers. 2001. 10. Nsibande, D., et al., Assessment of the uptake of neonatal and young infant referrals by community health workers to public health facilities in an urban informal settlement, KwaZulu-Natal, South Africa. BMC Health Serv Res, 2013. 13: p. 47.

African Health Sciences Vol 16 Issue 4, December, 2016
11. Waisswa P., P.S., Namazzi G., Ekirapa EK, The Uganda Newborn Study (UNEST): an effectiveness study on improving newborn health and survival in rural Uganda through a community-based intervention linked to health facilities - study protocol for a cluster randomized controlled trial. 2012.

12. Awasthi, S., et al., Effect of behaviour change communication on qualified medical care-seeking for sick neonates among urban poor in Lucknow, northern India: a before and after intervention study. Trop Med Int Health, 2009. 14(10): p. 1199-209.

13. Tripathy, P., et al, Community mobilisation with women's groups facilitated by Accredited Social Health Activists (ASHAs) to improve maternal and newborn health in underserved areas of Jharkhand and Orissa: study protocol for a cluster-randomised controlled trial. 2011.

14. Waisbord, S.R., T. Michaelides, and M. Rasmuson, Communication and social capital in the control of avian influenza: lessons from behaviour change experiences in the Mekong Region. Glob Public Health, 2008. 3(2): p. $197-$ 213.

15. Briscoe, C. and F. Aboud, Behaviour change communication targeting four health behaviours in developing countries: a review of change techniques. Soc Sci Med, 2012. 75(4): p. 612-21.

16. Mushi, A.K., et al., Development of behaviour change communication strategy for a vaccination-linked malaria control tool in southern Tanzania. Malar J, 2008. 7: p. 191. 17. Abroms, L.C. and E.W. Maibach, The effectiveness of mass communication to change public behavior. Annu Rev Public Health, 2008. 29: p. 219-34.

18. Aggleton, P., Behavior change communication strategies. AIDS Educ Prev, 1997. 9(2): p. 111-23.

19. Low-Beer, D. and R.L. Stoneburner, Behaviour and communication change in reducing HIV: is Uganda unique? Afr J AIDS Res, 2003. 2(1): p. 9-21.

20. Das, A. and M. Sarkar, Pregnancy-related health information-seeking behaviors among rural pregnant women in India: validating the Wilson model in the Indian context. Yale J Biol Med, 2014. 87(3): p. 251-62.

21.Banerjee, S.K., et al., Effectiveness of a behavior change communication intervention to improve knowledge and perceptions about abortion in Bihar and Jharkhand, India. Int Perspect Sex Reprod Health, 2013. 39(3): p. 142-51. 22. Parmar, V., Disseminating maternal health information to rural women: a user centered design framework. AMIA Annu Symp Proc, 2010. 2010: p. 592-6. 